\title{
Impact and Challenges of Coronavirus on Daily Life and Living: A Cloud Based Communication Assistant during the COVID-19Pandemic
}

\author{
Shahnawaz Ahmad ${ }^{1}$ | Nazim Ali Khan ${ }^{2}$ | Shabana Mehfuz ${ }^{3}$ \\ ${ }^{1}$ Department of Electrical Engineering, Jamia Millia Islamia, New Delhi. \\ ${ }^{2}$ Department of Mechanical Engineering, DCRUST (A State University), Murthal, Sonipat. \\ ${ }^{3}$ Department of Electrical Engineering, Jamia Millia Islamia, New Delhi.
}

To Cite this Article

Shahnawaz Ahmad, Nazim Ali Khan and Shabana Mehfuz, "Impact and challenges of Coronavirus on Daily Life and Living: ACloud Based Communication Assistant During the COVID-19Pandemic", International Journal for Modern Trends in Science and Technology, 6(9): 97-101, 2020.

Article Info

Received on 11-August-2020, Revised on 19-August-2020, Accepted on 29-August-2020, Published on 09-September-2020.

\section{ABSTRACT}

Introduction: The COVID-19, Coronavirus Disease 2019, emerged as a hazardous disease that led manycausalities across the world. Maintaining physical distancing, a critical task with minimum trips has become ahot trend nowadays. To keep up with patient care amidst an international crisis that is exhausting online

healthcare resources, physical distancing during the Covid-19 pandemic has brought telemonitoring to theforefront. Physical distancing measures are of utmost importance for understanding and managing health-relatedconcerns resulting.

Objectives: To describe and analyze the impact, challenges, and solutions of Coronavirus on daily life andlivingassociated with cloud based telemonitoring during the Covid-19 pandemic.

Methods: We presented a cloud-based communication assistant for the treatment of any customers withrecommender systems. Proposed Cloud-Based Communication Assistant (CBCA) aims first to identify thecustomer's query and then based on his/her need to provide better assistant at home. It categorized the customer'squery based on questionnaires. As customer register themselves online on the CBSCA in real time, it createsthe database for the same.

Results: This database helps to improve communication accuracy as it contains latest updates from realworldcases data. A team of shopkeepers, vendors, consultants are integrated with the CBCA for better consultationand prevention.

Conclusion: The ultimate aim of this proposed theory of CBCA is to take a control over unnecessary trips anddeaccelerate its rate of transmission among the society. Widespread adoption of telemonitoring-favoring policies are necessary and mostly needed to address mental health problems that may arise in areas of high infection and death rates.

KEYWORDS:Cloud Computing, Telemonitoring, Fuzzy Set Theory, Communication Medium, COVID-19 


\section{INTRODUCTION}

The COVID-19 formerly known as 2019-nCoV, severe acute respiratory syndrome coronavirus 2 , was firstfound in Wuhan in late 2019 as a case of untraceable pneumonia [1,2]. The World Health Organization named 2019-nCoV as COVID-19 (Coronavirus disease 2019) on February 11, 2020 [6]. COVID-19 was neitherdetected nor reported in humans before $[3,4]$.The reported cases of COVID-19 reached 27,296,207 as on September 07, 2020 [5]. COVID-19 is acommunicable disease which transmitted through close contact with the infected person or even with theinfected surface. As per the reports, it is highly contagious and period of its incubation can be 2 weeks or morethan that [6]. It infects the lung. Severely infected patients faced problem in respiration which leads to acuterespiratory distress syndrome and finally caused death $[7,8]$. Hence it is necessary to detect this problem at theearly stages so that its expansion can be controlled. Current viral nucleic testing of COVID-19 is having only

$30 \%-50 \%$ of accuracy. Due to this fact, many suspected or suspicious cases got unnoticed and undetectablewhich are left out from treatment [9]. Initially, "Diagnosis andTreatment Scheme for Pneumonia of COVID-19 (InterimVersion 5)" [10], was adopted by National HealthCommission of China, which was based on thechest imaging while proposing Version 6 [11]. Despite of adopting the aforesaid proposed Version 6, few casesstill remains untouched or misdiagnosed due to different level of diagnosis through different doctors in differentregions. Also, the detection of COVID-19 goes untraceable in its initial stage. Bernheim A et al. [12] revealedthat detection of nucleic acid test can be done by observing the changes in computed tomography (CT). It ismuch more important to diagnose the suspects and isolate them to prevent the further dispersion of the infectionamong the society. For this reason, we are proposing $\mathrm{CBCA}$ for unnecessary visits to shopping, working,transport, restaurant, and hospital. The CBCA will identify the customer's query and then based on his/herneed to provide better assistant at home. This will be really helpful for the customers who are not able tovisit any such places. It also prevents unnecessary visits to needy places in such hazardous scenario. With thehelp of the proposed method customers will be able to recommend themselves, because all the relevantconsultation will be easily identified by the proposed system.
Rest of the parts of this paper are as follows: Section II gives overview of cloud computing, Section III explainsFuzzy Set Theory, Proposed methodology is given by Section IV and finally, Section V will have conclusionsand research directions.

\section{CLOUD COMPUTING}

"Cloud computing (CC) is the on-demand delivery of compute power, database, storage, and other IT resourcesthrough cloud computing services via internet with go as you pricing model". Technology Review followed thecoinage of the term CC back two decades earlier, to November 14' 1996, and to an office park outside Houston

by "George Favaloro" and "Sean O'Sullivan" [13]. Eventhough many people believe that $\mathrm{CC}$ is a quite newbornphenomenon, it has it roots in the ideas assumed in 1960s. J.C.R. Licklider of ARPANET is universallyattributed as the first one to introduce the idea of "intergalactic computer network" in 1969, a machine whichcan be accessed from anywhere in the world. But even before him, in 1961, John McCarthy drifted the idea ofcomputation being provided as public service just like any other service, a concept he named as "utilitycomputing" [14], and in many ways, this is absolutely what CC is these days. Over the 1960's and 70's, largebanks of computers added so-called "time-sharing" services to local and remote stakeholders. In the 1980's and

early 90's, large scattered data centers became familiar place in large enterprises. There wasn't any momentousbreakthrough until Internet became quite common and easily accessible. Saalesforce.com in 1999 was the firstcompany to provide enterprise level applications to their customers through web.

\section{FUZZY SET THEORY}

Fuzzy set theory is developed by Lutfi. A. Zadeh in the year 1965. In order to use vague values, we use fuzzylogic. The range lies in fuzzy logic is [0-1]. There are several types of fuzzy set theory like as trapezoidal fuzzynumber, bell shaped fuzzy number, Gaussian fuzzy number, Triangular Fuzzy Number (TFN) $[15,16,17]$. Inour proposed method we are using triangular fuzzy number to calculate the weight of questionnaires based onDM's. Our proposed method will provide the optimal treatment to the COVID-19 victim at home through fuzzyassessment calculation by TFN. In fuzzy system, we can only provide the linguistic values like $(0,0.1,0.2,0.3,0.4,0.5,0.6,0.7,0.8,0.9,1)$ to 
each question based on DM's expertise. Once we get the linguistic values fromDM's, then we apply TFN to each DM's decision, and calculate the weight accordingly. MCDM (MultiCriterion Decision Making) method could be a sub-discipline of operations investigate that unequivocallyassesses numerous conflicting criteria in choice-making (both in existence and in settings such as commerce,government, and medication). In our proposed method, we are using fuzzy settheory for identifying the vaguevales from the questionnaires.

\section{PROPOSED METHODOLOGY}

This section presents a Cloud-Based Shopping Communication Assistant for the treatment of any

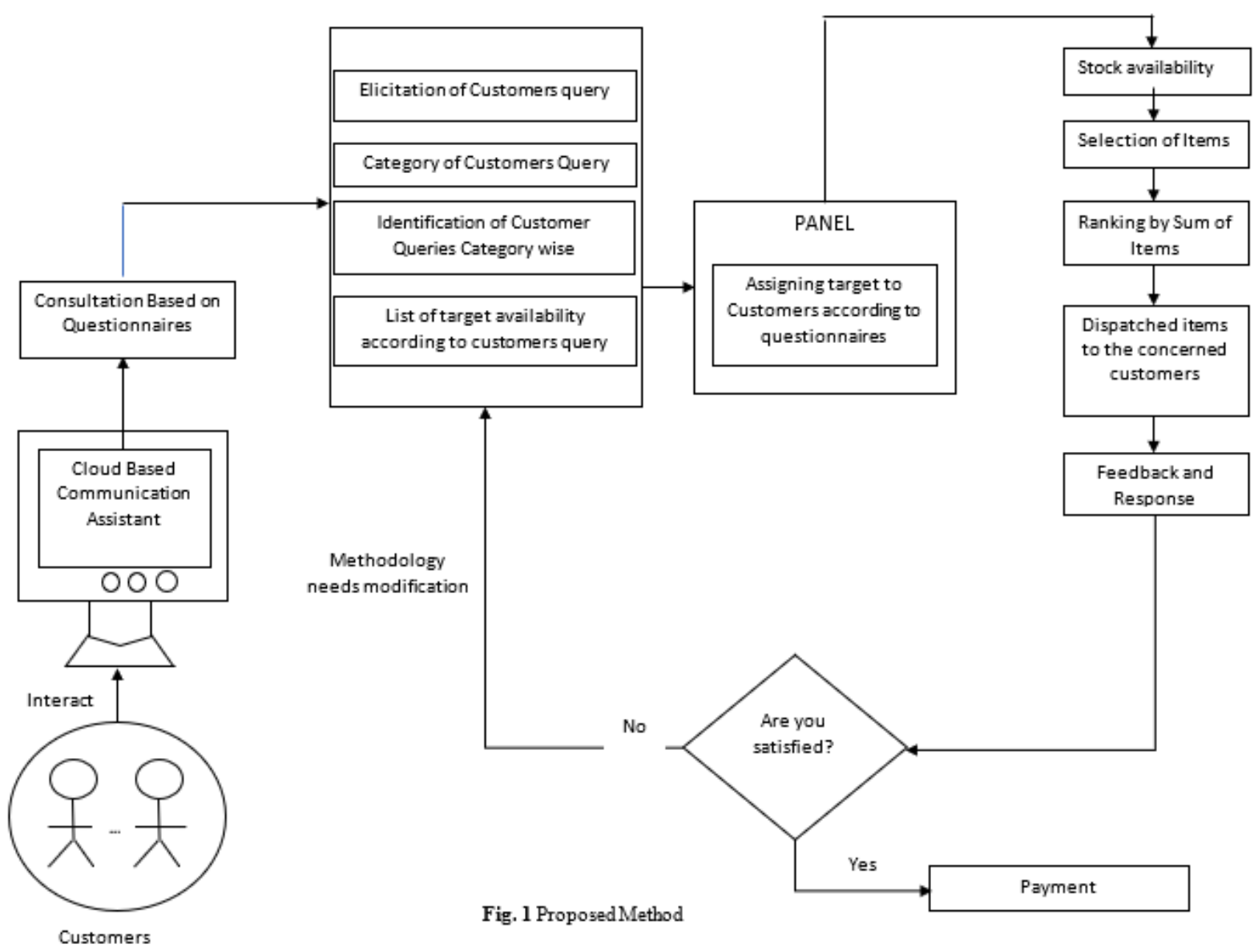

customerswith recommender systems. The proposed method is presented in the following (see Fig. 1)

(a) Register into CBCA.

(b) Consultation Based on Questionnaire by the Shopkeepers

(c) Identify customer for different regions

(d) Elicitation of customer's query

(e) Identification of customer's query category wise (f)Assigning target to Customers according to questionnaires

(g) Dispatched items to the concerned customers 
target and also provide the best possible assistance to the customers based on his/her query. This will be really helpful for the suspects who are not able to visit any shopping, working, transport, restaurant, and hospital. It also prevents unnecessary visits to hospitals in such hazardous scenario. With the help of the proposed method customers will be able to assist themselves, because all the relevant consultation will be easily identified by the proposed system. Future research directions include the following:

1. To extend the proposed method by using Multi-Criteria Decision-Making methods like TOPSIS, AHP, MAUT, CBR, DEA, SMART, Goal Programming, ELECTRE, PROMETHEE, SAW etc.; and to design a hybrid system by using an efficient method for mining frequency item sets.

2. To present the comparative study between various diagnosis center for getting cure from COVID-19.

\section{REFERENCES}

[1] http://www.chinacdc.cn/jkzt/crb/zl/szkb_11803/jszl_11 809/202001/t20200119_211274.html.

[2] http://m.news.cctv.com/2020/01/09/ARTI9Vp9Lra4Tvlt z3r7es96200109.shtml.

[3] LI Q, GUAN X, WU P, et al. Early transmission dynamics in Wuhan, China, of Novel coronavirus-infected pneumonia[J]. N Engl J Med, 2020 Jan 29. DOI: 10.1056/NEJMoa2001316. [Epub ahead of print])

[4] 4.https://www.who.int/publications-detail/clinical-mana gement-of-severe-acute-respiratory-infecti on-when-novel-coronavirus-(ncov)-infection-is-suspected.

[5] https://www.arcgis.com/apps/opsdashboard/index.html \#/bda7594740fd40299423467b48e9ecf6

[6] GUAN WJ, NI ZY, HU Y, et al. Clinical characteristics of 2019 novel coronavirus infection in China. medRxiv preprint http://doi.org/10.1101/2020.02.06.20020974.

[7] World Health Organization. Clinical management of severe acute respiratory infection when novel coronavirus ( $\mathrm{nCoV}$ ) infection is suspected. Interim guidance. 12 January 2020.

[8] Zhe Xu, Lei Shi, Yijin Wang, et al. Pathological findings of COVID-19 associated with acute respiratory distress syndrome. Lancet Respir Med. 2020. DOI: 10.1016/s2213-2600(20)30076-x.

[9] http://news.cctv.com/2020/02/07/ARTI9A303uk7NclMf BgjlAgx200207.shtml.

[10] National Health Commission of the People's Republic of China. Diagnosis and Treatment Scheme for Pneumonia of COVID-19 (Interim Version 5).

[11] National Health Commission of the People's Republic of China. Diagnosis and Treatment Scheme for Pneumonia of COVID-19 (Interim Version 6).

[12] Bernheim A, Mei X, Huang M, et al. Chest CT Findings in Coronavirus Disease-19 (COVID-19): Relationship to Duration of Infection. Radiology. 2020, 0(0): 200463.

[13] https://s3.amazonaws.com/files.technologyreview.com/p /pub/legacy/compaq_cst_1996_0.pdf.

[14] https://computinginthecloud.wordpress.com/2008/09/2 5/utility-cloud computingflashback-to-1961-prof-john-mccarthy/.
[15] Sadiq Mohd, "Prediction of Software Project Effort Using Fuzzy Logic", IEEE 3rd International Conference on Electronics, Computer Technology, pp. 353-358, 2014.

[16] Sadiq M and Jain S.K., "A Fuzzy Based Approach for Requirements Prioritization in Goal Oriented Requirements Elicitation Process", International Conference of Software Engineering and Knowledge Engineering (SEKE), pp. 54-58, 2013 USA.

[17] Shahnawaz Ahmad and Sadiq M, "Recommender Systems for Software Requirements Negotiation and Prioritization", International Journal of Computer Applications (09758887), Volume 117-No. 13, USA (New York), May 2015.

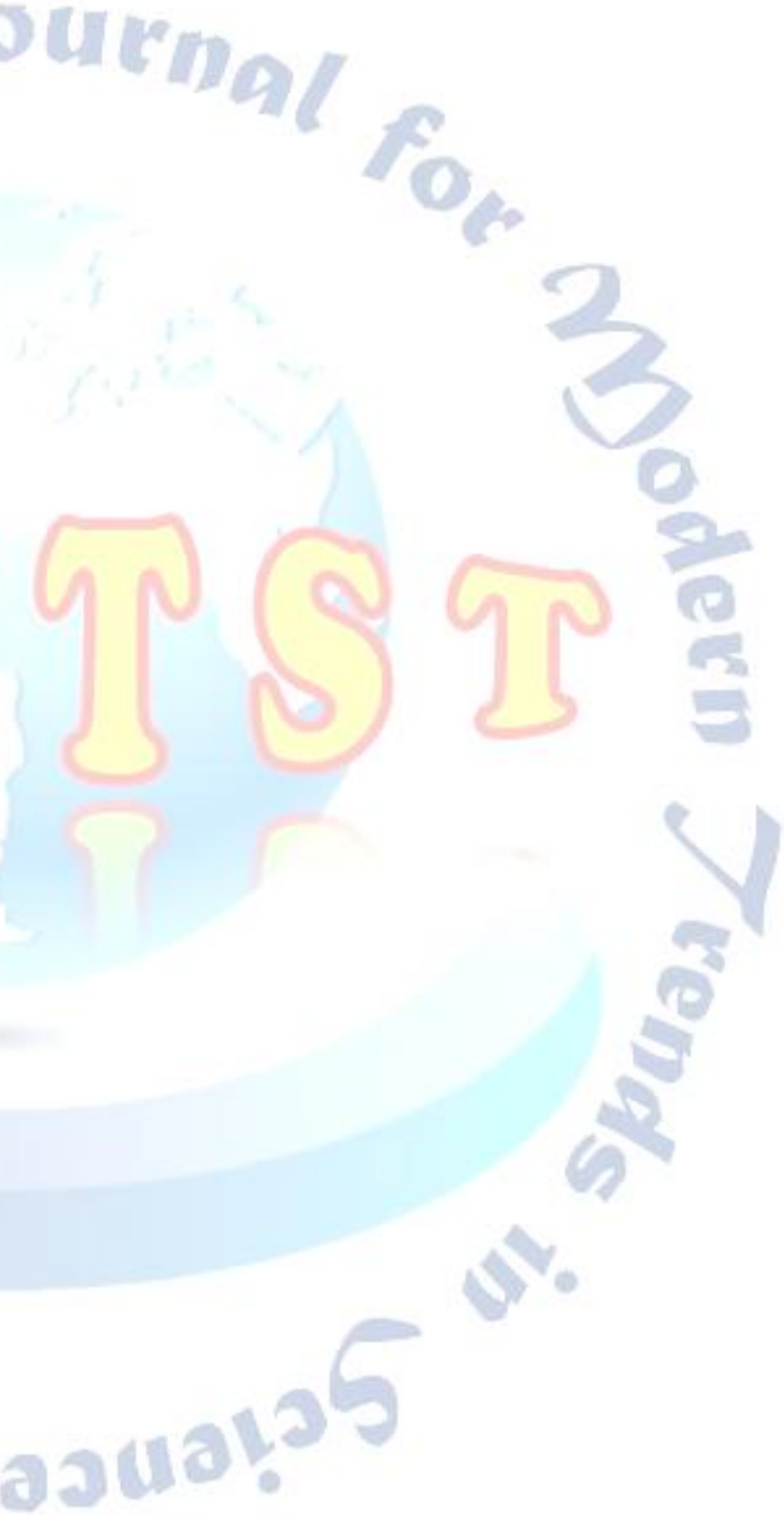

100 International Journal for Modern Trends in Science and Technology 


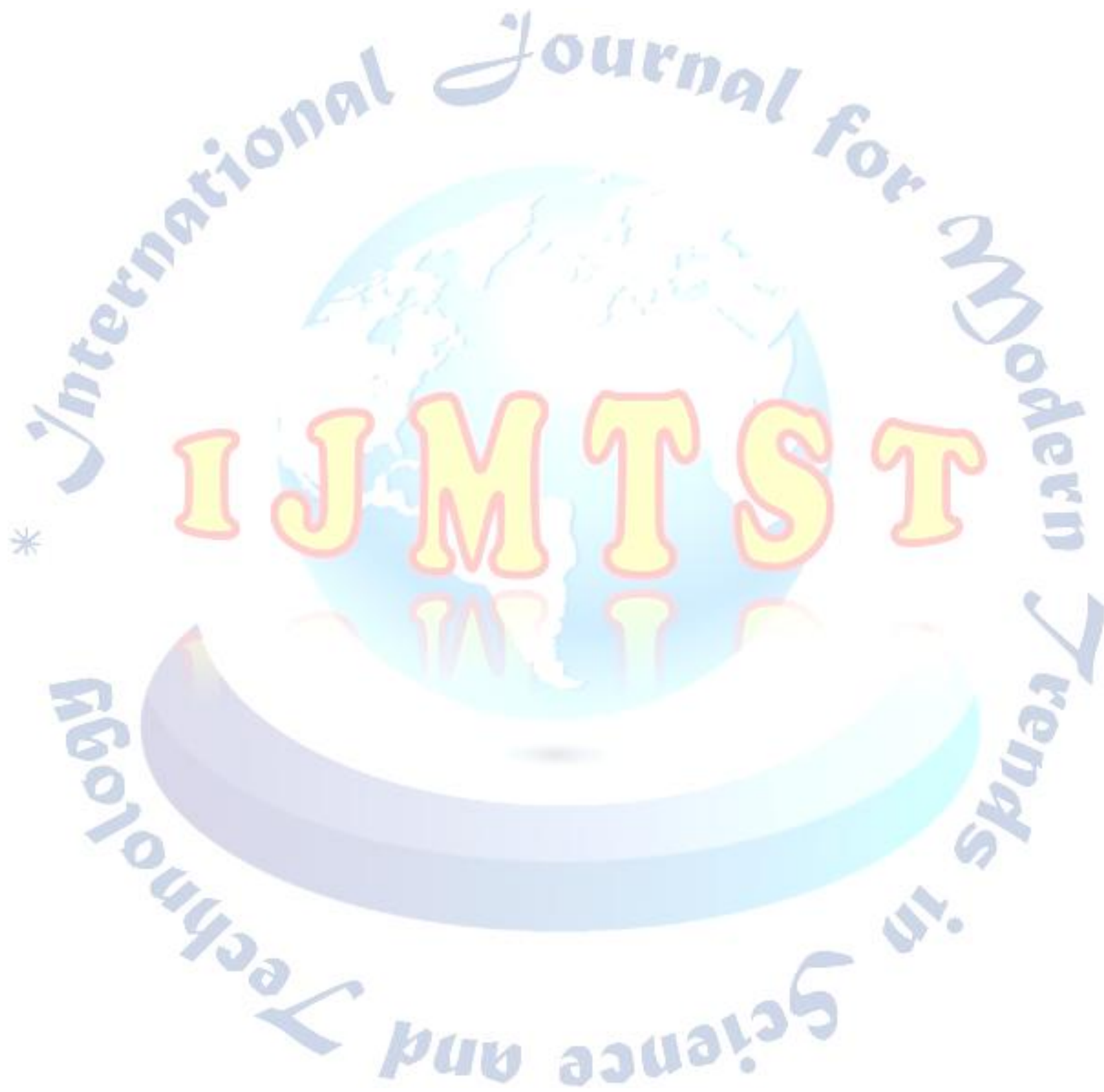

